Khan KS, Kunz R, Kleijnen J, et al. Systematic reviews to support evidencebased medicine: How to review and apply findings of healthcare research. London: Royal Society of Medicine Press, 2003.

Systematic reviews to support evidence-based medicine can be purchased online at www.rsmpress.co.uk/ for $£ 17.50$.
T his compact book attempts to guide the reader through the process of appraising and conducting a systematic review of the literature. It is divided into 3 sections: an introduction, the steps of a systematic review, and case studies. The introduction describes the goals of the book and how to use it. It provides a list of sources of systematic reviews and guidelines with relevant web addresses. The next section is divided into 5 chapters that address framing a question, identifying the relevant literature, assessing the quality of literature, summarising the evidence, and interpreting the findings. Each chapter explains how to appraise and conduct studies including discussion of key sources of potential bias. At the end of each chapter, key points about appraising and conducting systematic reviews are provided. For example, in the chapter describing identification of relevant literature, a l page summary illustrates examples of study selection criteria.

The application of the material discussed in the first section of the book is highlighted in section 3 using case studies. This section allows the reader to apply what was learned in the first section. Each case consists of a scenario requiring evidence from reviews, a demonstration of review methods, and a proposed resolution to the scenario. The topics for the case studies include identifying and appraising systematic reviews, reviewing evidence on the safety of a public health intervention, reviewing evidence on the effectiveness of therapy, and reviewing evidence on diagnostic test accuracy. The book also includes a glossary of useful terms and a list of additional resources that the reader can refer to.

Systematic reviews to support evidence-based medicine provides a concise overview of how to conduct and appraise a systematic review. However, it may not provide sufficient detail for the novice interested in conducting a systematic review and it may provide more details than many want when appraising a systematic review. It is very clearly written and illustrated with useful examples.

SHARON E STRAUS, MD, MSC, FRCPC DARLYNE RATH, MSC University of Toronto Toronto, Ontario, Canada

RATINGS:

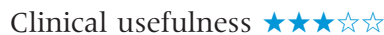

\title{
Please check your Bayes' nomogram!
}

S ome time ago, we inadvertently published a version of the Bayes' nomogram with ill spaced LRs. This appears to have had a virus-like effect, which we would ask you to help rectify by checking your notes, websites, etc. Ill formed versions will have the LR of 1000 and 0.001 at much greater spacing. If yours looks like this, please replace it with the one at www.cebm.net/likelihood ratios.asp which is "copy lefted." Ours thanks to Jan Hajek in the Netherlands for alerting us to the problem.

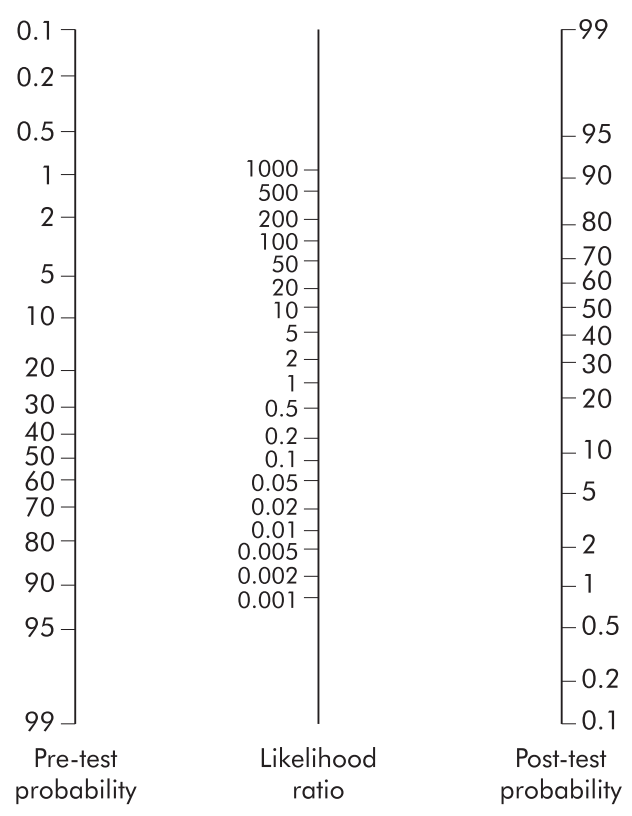

